\title{
Cenários para o futuro da educação a distância
}

Nairana Radtke Caneppele Bussler

Doutoranda em Administração na Universidade Nove de Julho UNINOVE. Programa de Pós Graduação em Administração - PPGA da UNINOVE

nacaneppele@ hotmail.com

Pang Lien Hsu

Doutorando pelo Programa de Pós-graduação em Administração - Estratégia - PPGA UNINOVE. Programa de Pós Graduação em Administração - PPGA da UNINOVE

panglienhsu@gmail.com

José Eduardo Storopoli

Doutor em Administração (Universidade Nove de Julho, UNINOVE. Programa de Pós Graduação em Administração - PPGA da UNINOVE

josees@uni9.pro.br

Emerson Antonio Maccari

Doutor em Administração pela USP com Estágio Doutoral na University of Massachusetts Amherst USA. Programa de Pós Graduação em Administração - PPGA da UNINOVE.

emerson.maccari@gmail.com

\section{Editor Científico: José Edson Lara}

Organização Comitê Científico

Double Blind Review pelo SEER/OJS

Recebido em 13.02.2019

Aprovado em 22.03.2019

Este trabalho foi licenciado com uma Licença Creative Commons - Atribuição - Não Comercial 3.0 Brasil 


\title{
Resumo
}

A evolução do uso da Educação a Distância $(\mathrm{EaD})$ é promovido a partir do avanço da tecnologia e, tem se mostrado como uma tendência para a educação nos próximos anos, além de ser uma oportunidade de negócio. O nosso objetivo neste estudo foi elaborar cenários para a EaD em Instituições de Ensino Superior (IES). Realizamos uma pesquisa bibliométrica utilizando o métodos de acoplamento bibliográfico, no qual extraídos utilizando o software VOSViewer, três clusters que proporcionaram a definição dos seguintes cenários: Comunidade de investigação, Interação e-learning e Ambientes de aprendizado combinado. Concluímos que a interpretação das tendências de estudos futuros transformadas em cenários para a $\mathrm{EaD}$ em IES, são úteis para a gestão desse modelo de educação que está em ascensão e que no futuro poderá dominar o método tradicional de ensino, mas os três cenários apresentados também evidenciaram que a interação não deixará de ser fator importante de satisfação.

Palavras-Chave: Ensino a Distância; Cenários; Educação Superior.

\section{Scenarios for the future of distance education}

\begin{abstract}
The evolution of the use of Distance Education (EAD) is promoted from the advancement of technology and has been shown as a trend for education in the coming years, in addition to being a business opportunity. Our objective in this study was to elaborate scenarios for EAD in Higher Education Institutions (IES). We performed a bibliometric research using the methods of bibliographic coupling, in which extracted using the VOSViewer software, three clusters that provided the definition of the following scenarios: Research community, Interaction e-learning and Environments of combined learning. We conclude that the interpretation of the trends of future studies transformed into scenarios for EAD in HEIs are useful for the management of this model of education that is on the rise and that in the future may dominate the traditional method of teaching, but the three scenarios presented also evidenced that interaction will not fail to be an important satisfaction factor.
\end{abstract}

Key words: Distance learning; Scenarios; College education.

\section{Cenarios para el futuro de la educación a distancia}

\section{Resumen}

La evolución del uso de la Educación a Distancia $(\mathrm{EaD})$ es promovida a partir del avance de la tecnología y, se ha mostrado como una tendencia a la educación en los próximos años, además de ser una oportunidad de negocio. Nuestro objetivo en este estudio fue elaborar escenarios para la EaD en Instituciones de Enseñanza Superior (IES). Realizamos una investigación bibliométrica utilizando los métodos de acoplamiento bibliográfico, en el que extraídos utilizando el software VOSViewer, tres clusters que proporcionaron la definición de los siguientes escenarios: Comunidad de investigación, Interacción e-learning y Ambientes de 
aprendizaje combinado. Concluimos que la interpretación de las tendencias de estudios futuros transformados en escenarios para la EaD en IES, son útiles para la gestión de ese modelo de educación que está en ascenso y que en el futuro podrá dominar el método tradicional de enseñanza, pero los tres escenarios presentados también evidenciaron que la interacción no dejará de ser un factor importante de satisfacción.

Palabras clave: Enseñanza a Distancia; escenarios; Educación universitaria.

\section{Introdução}

As matrículas do ensino superior no Brasil (presencial e a distância) tiveram uma expansão de apenas 3\% do total de alunos matriculados, nas duas modalidades, segundo dados do Censo da Educação Superior de 2017 realizado pelo Instituto Nacional de Estudos e Pesquisas Educacionais Anísio Teixeira (INEP). Esse aumento do número total e alunos foi possível em função do acréscimo de 17,6\% dos alunos matriculados na modalidade (EaD), o maior desde 2008, atendendo mais de 1,7 milhão de alunos, o que representa uma participação de $21,2 \%$ do total dos alunos de graduação no país. Já em relação às matrículas na modalidade presencial, houve uma queda de $0,4 \%$ de estudantes (INEP, 2017).

A EaD é considerada uma realidade para muitos estudantes com inúmero benefícios, porém, ela também apresentar uma sensação de isolamento o que pode ameaçar a capacidade do aluno aprender, pois, se não for bem conduzida e acompanhada, pode não proporcionar a devida interação entre os estudantes, o instrutor e os alunos, e entre alunos e conteúdo do curso (Moore, 1980; Bernard et al. 2009).

A EaD oferece oportunidades para não somente aumentar o alcance da educação e redução do seu custo, mas também para aumentar a qualidade do ensino e aprendizagem (Abrami, Bernard, Bures, Borokhovski \& Tamim, 2011). Por possuir diferentes pontos de vista sobre esse tema, e para avaliar o caminho a ser percorrido por essa nova forma de ensinar, autores como (Furrer \& Thomas, 2000; Ringland, 1998) sugerem o uso das técnicas de elaboração de cenários como ferramentas estratégicas importantes para fornecer visões confiáveis do futuro, melhorando a qualidade das decisões e aumentando a compreensão de suas implicações, oferecem ainda um meio de testar a viabilidade de estratégias alternativas, (Porter; Mannermaa, 1991).

Essas alternativas de futuros podem ser favoráveis ou desfavoráveis no ambiente de negócios (Geogantzas \& Acar, 1995), mas, ao construí-los, são veículos que auxiliam as pessoas a aprender pois são mais do que extrapolações das tendências presentes (Schwartz, 
2000), e " há evidências de que os métodos e as metodologias de construção de cenários se apresentam como um dos mais evoluídos instrumentos à disposição da Administração para o suporte e desenvolvimento de estratégias" (Boaventura, Costa \& Fischmann, p. 42, 2005).

O nosso objetivo neste estudo foi elaborar cenários para a educação a distância em Instituições de Ensino Superior, em virtude da evolução que esse apresentada por esse modelo de educação. Para tanto, realizamos uma pesquisa bibliométrica utilizando o métodos de acoplamento bibliográfico, no qual, utilizando o software VOSViewer 1.6.5, foram extraídos três clusters que proporcionaram a definição dos seguintes cenários: Comunidade de investigação, Interação e-learning e Ambientes de aprendizado combinado.

\section{Revisão da literatura}

\subsection{Cenários}

Os estudos, métodos e técnicas para identificação e planejamento com utilização de informações futuras pode ser denominado de diversas formas, tais como planejamento de cenários, desenvolvimento de cenários ou previsões, que podem ser resumidos de forma geral como estudos futuros (Dias, Vianna, \& Felby, 2016). Os cenários, um dos resultados produzidos pelos estudos futuros, podem ser considerados histórias que podem esclarecer sobre o futuro e que revelam o quão certo as variáveis do mercado e fatores ambientais serão no futuro, e não segue apenas a continuidade de tendências do passado (Dias et al., 2016; Wade, 2012).

Estudos já apontam que cenários são muito utilizados principalmente em áreas como ciências ambientais e ciências sociais, negócios, administração e contabilidade já que é uma ferramenta de planejamento que testa e mostra formas de melhoria no desempenho das organizações diante de ambientes dinâmicos (Oliveira, Barros, Pereira, Gomes, \& Costa, 2018).

Existem abordagens diferentes para realizar o planejamento de cenários prospectivos, elaborados por diversos autores, conforme já demonstrado por Oliveira et al. (2018), sejam eles quantitativos utilizando programas de simulação ou qualitativos utilizando a expertise a partir de entrevista de atores nas suas áreas específicas, há diversos pontos convergentes entre eles. Uma vez que todos eles são de algum modo, exercícios mentais com o produto final sob forma de cenários, criados baseado em critérios, informações e tendências, elas indicam com 
frequência de que a incerteza deve ser considerado como um ponto central na sua elaboração (Oliveira et al., 2018).

Portanto, cenários prospectivos contribuem para a tomada de decisão alargando o quadro de referência, permitindo que novas ideias e alternativas fluam, de forma que contribui para tornar mais claro as relações entre aspectos-chave que podem afetar as organizações (DIAS et al., 2016). A partir desse contexto, verifica-se que a utilização das técnicas de elaboração e cenários faz todo o sentido para se verificar a tendência do uso da educação a distância, o que permite melhorar a tomada de decisão sobre investimento neste tipo de modalidade de curso pelas organizações.

\subsection{Ensino a distância}

Ensino a distância $(\mathrm{EaD})$ é uma modalidade de ensino que já possui uma história bastante longa, datando antes da criação da internet, apesar do ensino online ter sido criado praticamente ao mesmo tempo do advento da web (Harasim, 2000). A procura por meios mais eficientes de ensino serviu como base para pesquisa e desenvolvimento em tecnologias educacionais, no início como uma forma de enriquecer a experiência de aprendizagem em sala de aula a partir da utilização de recursos audiovisuais nas primeiras décadas do século XX (Molenda, 2008).

O autor afirma que a criação da transmissão de sinais a rádio em meados de 1930 e da televisão nos anos de 1950 trouxeram uma mídia que conseguia atingir uma massa bem maior do público com programas de educativos. Sempre com o objetivo de ajudar as pessoas a aprenderem mais rápido, melhor e com um custo mais baixo, em meados de 1990 os computadores começaram a se tornarem o sistema de entrega educativa mais onipresente por causa da sua capacidade interativa e de processamento. A partir de 1995 com a intensificação do uso da internet, verificou-se uma forte expansão do uso do uso desta tecnologia para dar suporte ao ensino a distância (Molenda, 2008). Para que o EaD atinja o seu objetivo e tenha sucesso, certos fatores devem ser levados em conta, uma vez que essa modalidade difere do meio tradicional de ensino, tais fatores podem ser os ilustrados na Tabela 1, levantados por Storopoli (2016). 


\section{Tabela 1}

Fatores críticos de sucesso de EaD

\begin{tabular}{|c|c|}
\hline Fator & Fonte(s) \\
\hline Competência Docente & (Soong et al., 2001); (Selim, 2007) \\
\hline Atitude Docente & (Soong et al., 2001); (Selim, 2007) \\
\hline Colaboração & (Soong et al., 2001); (Selim, 2007) \\
\hline Suporte de plataforma & (Sun et al., 2008) \\
\hline Expectativa de Aprendizagem & (Sun et al., 2008) \\
\hline Flexibilidade & (Sun et al., 2008); (Liaw, 2008) \\
\hline Qualidade & (Sun et al., 2008); (Liaw, 2008) \\
\hline Pertinência & (Sun et al., 2008); (Liaw, 2008) \\
\hline Facilidade de Uso & (Sun et al., 2008); (Liaw, 2008) \\
\hline Diversidade de Conteúdos e Atividades & (Liaw, 2008) \\
\hline Satisfação & \\
\hline
\end{tabular}

Nota. Fonte: O uso do Knowledge discovery in database (KDD) de informações patentárias sobre ensino a distância: contribuições para instituições de ensino superior, de Storopoli, J. E. (2016), Universidade Nove de Julho - UNINOVE.

Todos esses fatores são necessários para que o $\mathrm{EaD}$ seja bem sucedida e que permita que os alunos e os professores se engajem nas plataformas de ensino à distância (Storopoli, 2016).

\section{Método e amostra}

Para essa pesquisa, nós buscamos artigos no banco de dados da Scopus sobre o tema de "higher education institutions" e "distance learning". Classificamos a natureza da pesquisa como qualitativa, com abordagem metodológica exploratória, através do paradigma interpretativista. Os métodos utilizados foram análise de conteúdo e bibliometria de artigos e revisões sobre o assunto.

O procedimento de coleta de dados foi estruturado através da busca por TITLE-ABSKEY ( "distance learn*" OR "Distance educat*" OR "distance degree*" OR "online educat*" OR "online learn*" OR "online degree*" OR "e-learn*" OR "virtual learn*" OR "virtual educat*" OR "virtual degree*" ) AND EXACTSRCTITLE ( "Higher Education" ) AND PUBYEAR > 2007 AND PUBYEAR < 2018 AND DOCTYPE ( ar OR re), no banco de artigos científicos Scopus, o que resultou em 615 artigos. 
Primeiro delimitamos os termos de pesquisa a serem utilizados para busca no banco de dados. Elas se resumiram em termos truncados das seguintes palavras: distance learning; distance education; distance degree; online education; online learning; virtual education e; virtual degree. Buscamos por esses termos no título, no resumo e também nas palavras-chave dos documentos.

O segundo recorte realizado foi a limitação dos periódicos pesquisados. Utilizando o código de busca "EXACTSRCTITLE" delimitamos apenas fontes com a palavra Higher Education como parte do seu título, uma vez que queremos apenas artigos que sejam específicos da área de ensino superior. O terceiro recorte se refere ao ano pesquisado, que foi publicações desde 2007 a 2018 totalizando documentos dos 10 últimos anos publicados sobre o assunto na área pesquisada. O último recorte realizado foi o tipo de documento recuperado pelos termos da pesquisa, que foram limitados à somente artigos científicos e revisões, pois esses dois tipos de documentos mais relevantes para servir como base de análise de HEI IN ENGAGING IN DISTANCE LEARNING, que é o objetivo deste artigo.

\section{Resultados}

A partir de da amostra de 615 artigos realizamos uma análise bibliométrica com o objetivo de extrair clusters da amostra e caracterizá-los como cenários do futuro da educação a distância no ensino superior (Figura 1).

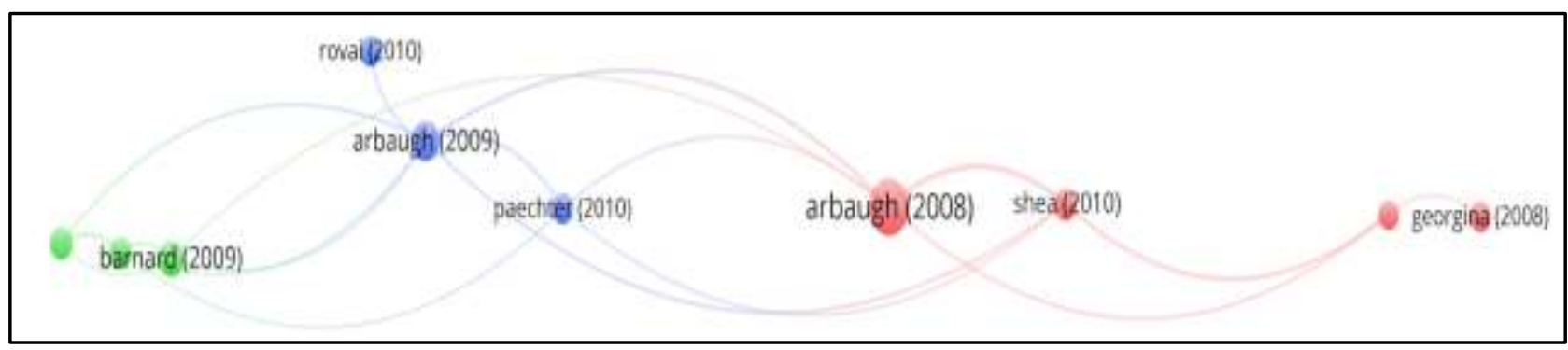

Figura 1. Clusters dos documentos do acoplamento bibliográfico

Com a base de dados extraídos, utilizamos o software VOSViewer 1.6.5 para realizar a análise de pareamento, da qual, para extrair os 3 clusters ilustrados na Figura 1, forçamos apenas a visualização de artigos com 80 citações ou mais. Com essa seleção, extraímos o total de 10 artigos divididos em 3 clusters. O primeiro cluster em denominamos de Comunidade de 
investigação, o segundo cluster foi nomeado como Interação e-learning, e o terceiro cluster foi denominado de Ambientes de aprendizado combinado.

\subsection{Cenário 1 - Comunidade de Investigação}

O conceito de Comunidade de Investigação tem suas raízes na teoria da aprendizagem sociocognitiva, especialmente na zona de desenvolvimento proximal de Vygotsky (ZDP) (Vygotsky, 1978). Nessa teoria, os indivíduos são capazes de realizar níveis intelectuais mais altos quando solicitados a trabalhar em situações colaborativas do que quando solicitados a trabalhar individualmente e, tanto a interação professor-aluno, quanto a interação aluno-aluno são importantes no processo de aprendizagem. As interações entre professor e alunos servem como "andaimes" e são definidas como uma "forma vicária de consciência, até que o aluno esteja apto a controlar de forma consciente e independente a nova função ou sistema conceitual sendo utilizado" (Bruner, 1985, p. 12); e as interações entre os estudantes entre si é uma colaboração caracterizada como uma "transação social [...], veículo fundamental para a educação" (Bruner, 1985, p. 15).

No início dos anos 1970, Matthew Lipman publica vários romances filosóficos para crianças, intitulado Filosofia para crianças (Lipman, 1976), e estabelece o Instituto para o Avanço da Filosofia para Crianças (IAPC). Seu principal objetivo era criar um ambiente de sala de aula propício à interação entre pares, com ênfase principal no diálogo e reflexão. Argumentase que as habilidades de pensamento crítico são melhor desenvolvidas dentro do contexto de diálogo de grupo sobre questões filosóficas (Lipman, 1991) e descoberta intelectual e compreensão são aumentadas por meio de diálogos, que são empregados desde Platão e Sócrates na Grécia no século VI a.C. O estabelecimento desse ambiente de sala de aula, definido como Comunidade de Investigação, começa com: (1) uso de critérios - encorajamento para examinar e explicar por que eles pensam como eles fazem sobre assuntos em discussão; (2) autocorreção - os indivíduos são encorajados a ouvir atentamente os comentários de cada membro da comunidade e estar dispostos a reconsiderar seus próprios julgamentos e opiniões; e (3) atenção ao contexto - a compreensão da influência importante do contexto está no julgamento de uma pessoa e as opiniões são valorizadas (Hagaman, 1990).

Neste cenário, a ênfase está no ambiente em que a comunidade de investigação emerge, o qual possui alguns comportamentos característicos, tais como: os membros solicitam, uns dos 
outros, razões de crença; constroem as idéias uns dos outros; deliberam entre si; apontam possíveis contra-exemplos para as hipóteses dos outros; utilizam critérios específicos ao julgar; questionam um ao outro; e cooperam no desenvolvimento de técnicas racionais de resolução de problemas (Lipman, 1991). Esses comportamentos só podem ocorrer quando três elementos, essenciais para uma transação educacional, são estabelecidos.

O primeiro elemento é a presença cognitiva, mais básica para o sucesso, ela é definida como "a medida em que os participantes em qualquer configuração particular de uma comunidade de investigação são capazes de construir significado através da comunicação sustentada" (Garrison, Anderson, \& Archer, p. 89, 2000). O segundo elemento, denominado presença social, tem a importância primordial de funcionar como suporte para a presença cognitiva, facilitando indiretamente o processo de pensamento crítico realizado pela comunidade de aprendizes, e sua definição é “a capacidade dos participantes da Comunidade de Inquérito projetar suas características pessoais na comunidade, apresentando-se assim aos outros participantes como pessoas reais" (Garrison et al., p. 89, 2000). A presença de ensino constitui o terceiro elemento e consiste em duas funções gerais, que podem ser realizadas por qualquer participante de uma Comunidade de Inquérito. No entanto, em um ambiente educacional, essas funções provavelmente são a principal responsabilidade do professor.

A primeira dessas funções é o design da experiência educacional com a seleção, organização e apresentação primária do conteúdo do curso, concepção e desenvolvimento de atividades de aprendizagem e, em última instância, avaliação. A segunda função, facilitação, é uma responsabilidade que pode ser compartilhada entre o professor e alguns ou todos os outros participantes ou estudantes. A presença de ensino também apoia e aumenta a presença social e cognitiva com o propósito de obter resultados educacionais e tem um papel fundamental no estabelecimento e sustentação de uma comunidade de investigação (Garrison, Cleveland-Innes, \& Fung, 2009).

A comunidade de investigação foi inicialmente concebida para a educação infantil, com uma taxa de sucesso substancial. Em uma revisão crítica sistemática de estudos de resultados controlados em escolas de ensino fundamental e médio, não houve resultados negativos de seu impacto (Trickey \& Topping, 2004). Mas desde então, a estrutura da comunidade de pesquisa se expandiu para outros domínios.

A primeira aplicação em aprendizes adultos desenvolveu o pensamento crítico através da fusão e harmonização da resolução de problemas e do pensamento criativo (Garrison, 1991). $\mathrm{O}$ conceito foi então aplicado ao ensino à distância e, embora utilizando um contexto de 
teleconferência de áudio, os resultados mostraram que ele estimulou a criação de novos conhecimentos integrados ao contexto de trabalho do professor e alunos, e os resultados são consistentes com um modelo de educação que enfatiza a aprendizagem profunda ou significativa que é consistente com o pensamento crítico (Anderson \& Garrison, 1995). Também foi aplicado em educação mediada por computador (Garrison et al., 2000) e em cursos online relacionados a negócios (Daspit \& D'Souza, 2012). Há evidências de que a estrutura da comunidade de investigação pode ser mais aplicável às disciplinas aplicadas, pois ela pressupõe uma abordagem construtivista do ensino e da aprendizagem, portanto, pode não se alinhar às abordagens cumulativas orientadas para o instrutor particularmente associadas a disciplinas duras e puras (Arbaugh, Bangert \& Cleveland-Innes, 2010).

Em todas essas experiências, apenas a interação não é suficiente para alcançar níveis mais altos ou aprendizado (Garrison \& Cleveland-Innes, 2005). A presença de ensino permite abordagens profundas ao aprendizado, mesmo quando a presença de ensino significa que o aprendizado médio foi aprimorado. E sempre que a presença de ensino era baixa, apesar dos altos níveis de interações, o aprendizado não era aprimorado. Assim, a presença de ensino e a natureza das interações são cruciais para abordagens profundas de aprendizagem (Garrison et al., 2009; Borup, West, \& Graham, 2012).

As medidas adotadas para avaliação da Comunidade de Investigação, presença de ensino, social e cognitiva podem se sobrepor conforme evidenciado pela correlação entre os fatores no estudo de Arbaugh, Cleveland-Innes, Diaz, Garrison, Ice, Richardson, \& Swan (2008). Para os autores, as descobertas sugerem que os instrutores desempenham papéis significativos e os alunos entendem e aplicam a conduta apropriada dentro do curso antes de começar e orientar as atividades mesmo que o curso esteja em andamento.

\subsection{Cenário 2 - Interação e-learning}

A literatura sobre o $\mathrm{EaD}$ e o e-learning admitem somente uma interpretação sobre a importância da interação entre eles (Anderson 2003; Sutton 2001; Sims 1999; Wagner 1994; Jaspers 1991; Moore 1989), pois presume-se que a interação entre alunos, professores e conteúdo foi em grande parte ausente durante o início da história (Nipper 1989). Compreendendo a interação como importante em todas as formas de educação, admite-se que ela proporciona que alunos possam vincular conhecimento preexistente a novas informações e 
fazer um novo significado através de análise ou integração, estando relacionada com a qualidade da aprendizagem online (Han \& Johnson, 2012; Juwah, 2006).

A evidência empírica da importância da interação foi realizada por Bernard, Abrami, Borokhovski, Wade, Tamim \& Surkes (2009) evidenciando 74 estudos empíricos comparando diferentes modos de $\mathrm{EaD}$ e o e-learning entre si, o qual resultou em o três tipos de interações: entre estudantes, entre o instrutor e os alunos e entre os alunos e o conteúdo do curso. A primeira refere-se à interação entre alunos individuais ou entre os alunos que trabalham em pequenos grupos. Já a interação entre o aluno e o instrutor acontece quando o instrutor procura estimular ou manter o interesse do aluno no que é ensinado, para motivando-o a aprender, o instrutor inclui auto-orientação e auto-motivação. A interação entre os alunos e o conteúdo do curso é o processo de interagir intelectualmente com o conteúdo que resulta em mudanças na compreensão, perspectivas e estruturas cognitivas do aprendiz (Moore, 1989). Outras formas de interação também foram propostas, como a interação aluno-designer, aluno-tutor (Juwah, 2006), interação aluno-interface (Gunawardena, Lowe, \& Anderson, 1997), interação alunoferramenta (Hirumi, 2011), aluno-interação de tarefas (Herrington, Reeves, \& Oliver, 2006), e interação vicária (Sutton, 2001).

Para que essas interações sejam colocadas em prática, tanto na $\mathrm{EaD}$ quanto no elearning, não devem mais ser comparadas como instruções em sala de aula, pois possuem um novo design no qual formas de evidência podem melhorar a prática instrucional (Abrami, Bernard, Bures, Borokhovski \& Tamim, 2011). Para os autores, avanços mais recentes em tecnologia aumentaram o poder, a flexibilidade e a onipresença, facilitando todos os processos.

$\mathrm{O} \mathrm{EaD}$ e o e-learning eliminam a limitação de lugar, tempo e materiais físicos e em grande medida dá aos alunos o controle sobre quando, o que e como estudar (Cunningham \& Billingsley, 2003). Alunos com as crenças de responsabilização internos geralmente têm melhor desempenho do que os estudantes com um locus de controle externo em cursos online (Bowen, 1996).

Portanto, essa forma de ensinar e aprender oferecem oportunidades para aumentar o alcance da educação e reduzir seu custo se for realizada com qualidade. Compreender o contexto, a forma de utilização, sua eficiência e eficácia perante a aprendizagem dos alunos, proporcionam uma mudança fundamental na cultura das práticas de pesquisa (Cook, 2009), e embora as tecnologias emergentes proporcionam várias oportunidades que geram a colaboração em ambientes de aprendizagem, os programas de $\mathrm{EaD}$ em todo o mundo enfrentam desafios que podem limitar ou implementação destas tecnologias (Beldarrain, 2006). 
Algumas práticas podem ser utilizadas para enfrentar esses desafios as quais precisam de uma interação intencional entre todas as partes envolvidas. Proporcionar que o aluno seja auto-regulado, estabelecendo metas claras para desenvolver estratégias para alcançar objetivos, monitorando suas atividades para verificar suas realizações usando e feedback de colegas ou professores pode ser uma estratégia (Zimmerman 2008). A segunda está relacionada à ciência do processo de aprendizagem a ser realizado, onde esse processo conduz, e porque a aprendizagem é importante (Knowles, 1980).

Essas abordagens colocam ênfase no individual, e pesquisadores têm mostrado os efeitos de comportamentos de auto-regulamentação sobre o desempenho acadêmico dos alunos em salas de aula regulares (Lan, 1996; Kramarski \& Mizrachi, 2006; Kramarski \& Gutman, 2006) pois como o número de alunos matriculados em cursos on-line está aumentando, é provável que a variação nos métodos de ensino e o aprendizado que ocorrer também aumentará, compreendendo que esses cursos estão expandindo-se além do ambiente educacional tradicional (Barnard, Lan, To, Paton, \& Lai, 2009).

A reconciliação com mais de uma perspectiva sócio construtivista não necessariamente proíbe o conceito de auto-regulação, mas é apresentada por Hickey e McCaslin (2001) como forma de enquadramento no contexto dos estudantes pois aumenta o envolvimento destes nas práticas. Utilizar princípios e teorias de psicologia educacional são necessários para avaliar as características únicas do ambiente de aprendizagem auxiliando em sua compreensão (Broad, 1999 ). Mesmo assim, se os desafios se intensificarem, torna-se relevante compreender que os alunos precisam de mais orientações sobre as ferramentas, também precisam praticar para usálas com sabedoria, e observar que as ferramentas cognitivas e estratégias de aprendizagem podem funcionar melhor quando são característica de um curso (Abrami et al., 2011).

Outro ponto importante a ser ressaltado quando se aborda a interação, está relacionado a satisfação dos alunos na avaliação de cursos de $\mathrm{EaD}$, uma vez que está relacionado com a qualidade dos programas e desempenho dos alunos, sendo a interação um indicador crítico de satisfação dos alunos (Kuo, Walker, Schroder \& Belland, 2014). Os autores testaram um modelo de regressão para verificar a satisfação do aluno envolvendo características do estudante e três tipos de interação, auto-eficácia da Internet, aprendizagem auto-regulada e preditores de nível de classe, tais como categoria de curso e programa acadêmico). A conclusão que se chegou é que as melhorias relacionadas ao aluno, como o rendimento da interação de conteúdo, é mais 
promissor para aumentar a satisfação do deste, e essa interação aluno com aluno pode ser insignificante nas configurações do curso on-line.

\subsection{Cenário 3 - ambiente de aprendizado combinado}

Durante a realização de estudos, alguns avanços em diferentes disciplinas são ignorados, em parte pois pesquisadores de uma disciplina desconhecem as perspectivas teóricas e estruturas conceituais de disciplinas relacionadas que poderiam auxiliar a explicar os fenômenos em sua própria disciplina (Wan, Fang \& Neufeld, 2007; Bryant, Kahle, \& Schafer, 2005). Outro fator está relacionado aos avanços nas abordagens metodológicas e analíticas utilizados em uma disciplina por vezes não são compartilhadas, gerando uma qualidade de pesquisa amplamente variável entre disciplinas, onde pode inexistir evidência de motivação para tomada de decisão (Arbaugh, Godfrey, Johnson, Pollack, Niendorf, \& Wresch, 2009; Arbaugh \& Warell, 2009).

Com base na análise de disciplinas e desenhos didáticos de cursos compreende-se que diferentes campos de instrução podem ser observados, como é o caso da Ead e da educação presencial. O desenvolvimento de sistemas de garantia de qualidade que descrevam as características desejáveis, bem como modelos instrucionais distinguem os campos de instrução em resultados de aprendizagem, desenho do curso, material didático e ambiente do curso eletrônico, interação entre alunos e um instrutor, interação com estudantes de pares, e processos individuais de aprendizagem são importantes para a identificação da efetividade e dos avanços dos campos (Brophy, 1999; Ehlers, 2004; Young \& Norgard, 2006).

O ensino tradicional, aquele realizado em sala de aula com professores e alunos assistindo a aula de forma presencial, é um modelo que já está enraizado, já o Ead requer uma grande quantidade de apoio para que se torne parte do perfil das instituições (Berge, 2007). Para o autor, isso só acontecerá se for resultado de uma mudança na avaliação da educação, a qual valorize a aprendizagem, o planejamento estratégico e a garantia da qualidade.

Avaliando alguns estudos para descrição deste cenário, podemos observar que as percepções sobre a $\mathrm{EaD}$ e o ensino presencial de certa forma se completam, interagindo com estratégias e metodologias que façam o aluno pertencer ao processo. Como exemplo é possível citar o estudo de Arbaugh et al. (2009), que avaliaram ambientes mediados por tecnologia, e verificaram que a participação entre os grupos foi mais democratizado e eles poderiam desenvolver relações colaborativas produtivas com seus companheiros de equipe, fato esse que 
foi possível em função de fatores de maturidade profissional dos estudantes que influenciam a eficácia de tais ferramentas.

No entanto, apesar de não existir diferenças entre os dois meios em termos de desempenho, existem sim diferenças significativas relacionadas às atitudes dos alunos em relação à internet como um meio de entrega (Sankaran, Sankaran, \& Bui, 2000; Piccoli, Ahmad, \& Ives, 2001; Abraham, 2002). Em contraponto, Sankaran e Bui (2001) verificaram que a diferença de desempenho são atribuídas às estratégias de aprendizagem e motivação e não as características do meio de entrega.

Por outro lado, Piccoli, Ahmad \& Ives (2001) identificaram que, embora os alunos do $\mathrm{EaD}$ possuam mais confiança na sua própria capacidade para completar uma determinada tarefa ou resolver um problema e estudar, eles estavam menos satisfeitos com as experiência do curso como um todo. O que não se confirmou no estudo de Heckman e Annabi (2005), visto que os autores identificaram que os alunos nas discussões $\mathrm{EaD}$ tendem a ter muito mais responsabilidade pela criação do ambiente cognitivo e social do que quando trabalham em sala de aula de forma presencial, eram mais propensos a demonstrar processos cognitivos de ordem superior como resultado. $\mathrm{Na} \mathrm{EaD}$ os alunos eram muito mais propensos a responder uns aos outros depois de postar as respostas para o instrutor, enquanto os alunos em sala de aula apenas respondiam ao instrutor.

Em cursos com sala de aula combinada verificou-se que houve melhor desempenho nos exames observando o todo, mas os alunos, de forma individual, trabalham de forma mais efetiva em projetos de grupos e atribuições baseadas em atividades (Bryant, Campbell \& Kerr, 2003). Ao comparar ambientes de aprendizagem combinados Klein, Noe e Wang (2006) descobriram vários alunos com alta orientação para objetivos de aprendizagem, compreendendo o ambiente como local que permitisse, em vez de uma barreira, maior motivação para aprender. Eles observaram nestes ambientes os alunos com mais controle, foram desafiados a apresentar um papel mais ativo em sua aprendizagem, o que facilitou a motivação para aprender mais do que instrução de sala de aula.

Diferentes contextos apresentaram diferentes resultados. Sitzmann, Kraiger, Stewart \& Wisher (2006) descobriram que o ensino EaD foi $6 \%$ mais eficaz do que o ensino presencial, mas Al-Shammari (2005) identificou que a colaboração no EaD era significativamente menos eficaz para o desenvolvimento de competências baseadas no conhecimento. No estudo de Paechter \& Maier (2010) os alunos apreciaram o EaD para o seu potencial em fornecer uma 
estrutura clara e coerente do material de aprendizagem, no apoio à aprendizagem auto-regulada, e na distribuição de informação. Para fins de comunicação em que um entendimento comum tem de ser derivado ou em que as relações interpessoais devem ser estabelecidas, eles preferiram o ensino presencial, pois quando o conhecimento conceitual no assunto ou habilidades na aplicação deste conhecimento estão a ser adquirido, os alunos preferem aprendizagem presencial. No entanto, quando as habilidades de aprendizagem auto-regulada estão a ser adquirido, os alunos defendem o EaD.

$\mathrm{Na} \mathrm{EaD}$ os instrutores possuem um papel fundamental, eles auxiliam os alunos a aprender uns com os outros, fazendo com que os mesmos assimilem o ambiente e fatores de aprendizagem, sendo identificado como potencial e generalizável para aprendizagem combinada (Walker, 2003; Proserpio \& Gioia, 2007). Para tanto, a utilização de um modelo geral de eficácia da aprendizagem como uma função de fatores de conteúdo, fatores relacionados à entrega e fatores de aprendizagem pode ser adotado para a geração de um desempenho ainda mais favorável (Rungtusanatham, Ellram, Siferd \& Salik, 2004). Para os autores, estes fatores geraram quatro modelos de $\mathrm{EaD}$ sendo dois tipos de modelos de visão geral do conteúdo, um modelo de habilidades técnicas e um modelo de aprendizagem gerencial.

Hollenbeck, Zinkhan e French (2005) também desenvolveram um modelo com etapas para a implementação de $\mathrm{EaD}$, o qual está focado em fornecer tecnologia confiável, construir comunidades entre os alunos, incentivando professores a ensinar no programa como blocos iniciais de construção. Para os autores, é importante a efetiva atuação dos coordenadores do programa, pois eles necessitam garantir a comunicação adequada no processo de planejamento, a interação entre alunos e corpo docente e a implementação do processo de avaliação, gerando um ambiente de sala de aula com recursos combinados.

\section{Discussão}

Neste estudo o nosso objetivo neste estudo foi elaborar cenários para a educação a distância em Instituições de Ensino Superior em virtude da evolução que esse apresentada por esse modelo de educação. Para tanto, realizamos uma pesquisa bibliométrica utilizando o métodos de acoplamento bibliográfico, no qual extraídos, utilizando o software VOSViewer 1.6.5, resultando em três clusters que proporcionaram a identificação dos cenários apresentados na Tabela 2. 
Tabela 2.

Cenários desenvolvidos a partir de um estudo bibliométrico sobre o $\mathrm{EaD}$

\begin{tabular}{|c|c|c|}
\hline Cenário & Premissas & Autores \\
\hline $\begin{array}{l}\text { COMUNIDADE DE } \\
\text { INVESTIGAÇÃO }\end{array}$ & $\begin{array}{ll}\text { - } & \text { Presença cognitiva } \\
\text { - } & \text { Presença social } \\
\text { - } & \text { Presença de ensino } \\
\text { - } & \text { Comunicação assíncrona } \\
\text { - } & \text { Ensino híbrido } \\
\text { - } & \text { Integração e treinamento } \\
& \text { através da tecnologia }\end{array}$ & $\begin{array}{l}\text { Arbaugh et al (2008), Borup, West, \& Graham } \\
\text { (2012), Georgina \& Olson (2008), Shea, et al } \\
\text { (2010), Vygotsky (1978), Bruner (1985), } \\
\text { Lipman (1976: 1991), Hagaman (1990), } \\
\text { Garrison, Anderson, \& Archer (2000), } \\
\text { Garrison, Cleveland-Innes, \& Fung, (2009), } \\
\text { Trickey \& Topping, (2004), Anderson \& } \\
\text { Garrison, (1995), Daspit \& D'Souza, (2012), } \\
\text { Arbaugh, Bangert, \& Cleveland-Innes (2010). }\end{array}$ \\
\hline $\begin{array}{l}\text { INTERAÇÃO } \\
\text { E-LEARNING }\end{array}$ & 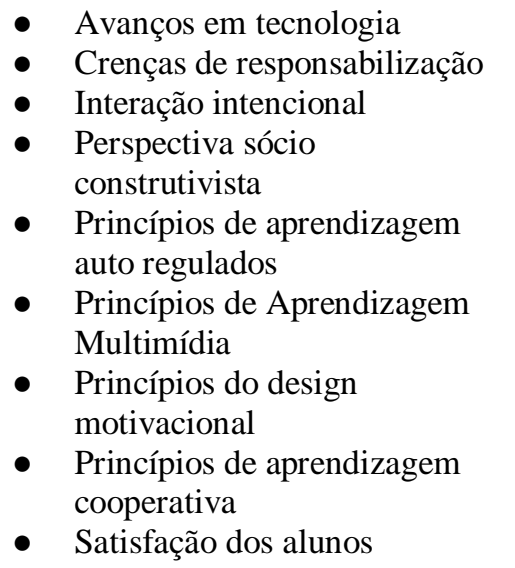 & $\begin{array}{l}\text { Anderson (2003), Sutton (2001), Sims (1999), } \\
\text { Wagner (1994), Jaspers (1991), Moore } \\
\text { (1989), Nipper (1989), Han \& Johnson (2012), } \\
\text { Juwah (2006), Bernard et al. (2009), Moore } \\
\text { (1989), Gunawardena, Lowe, \& Anderson } \\
\text { (1997), Hirumi (2011), Herrington, Reeves, \& } \\
\text { Oliver (2006), Sutton (2001), Abrami et al. } \\
\text { (2011), Cunningham \& Billingsley (2003), } \\
\text { Bowen (1996), Cook (2009), Beldarrain } \\
\text { (2006), Zimmerman (2008), Knowles (1980), } \\
\text { Lan (1996), Kramarski \& Mizrachi (2006), } \\
\text { Kramarski \& Gutman (2006), Barnard et al. } \\
\text { (2009), Hickey \& McCaslin (2001), Broad } \\
\text { (1999), Kuo et al. (2014). }\end{array}$ \\
\hline $\begin{array}{c}\text { AMBIENTE DE } \\
\text { APRENDIZADO } \\
\text { COMBINADO }\end{array}$ & $\begin{array}{l}\text { - } \text { Abordagens metodológicas } \\
\text { - } \text { Ensino tradicional } \\
\text { - } \text { Ead } \\
\text { colaçõos } \\
\text { - } \text { Maturidade profissional } \\
\text { - } \text { Eficácia das ferramentas. } \\
\text { - Atitudes } \\
\text { dos alunos } \\
\text { - Criação } \\
\text { do ambiente cognitivo e social } \\
\text { Objetivos } \\
\text { de aprendizagem } \\
\text { Desenvolvimento } \\
\text { de competências } \\
\text { Fatores de conteúdo, } \\
\text { relacionados à entrega e de } \\
\text { aprendizagem }\end{array}$ & $\begin{array}{l}\text { Wan, Fang \& Neufeld (2007), Bryant, Kahle, } \\
\text { \& Schafer (2005), Arbaugh et al. (2009), } \\
\text { Arbaugh \& Warell (2009), Brophy (1999), } \\
\text { Ehlers (2004), Young \& Norgard (2006), } \\
\text { Berge (2007), Sankaran, Sankaran \& Bui } \\
\text { (2000), Piccoli, Ahmad \& Ives (2001), } \\
\text { Abraham (2002), Sankaran \& Bui (2001), } \\
\text { Piccoli, Ahmad \& Ives (2001), Heckman \& } \\
\text { Annabi (2005), Bryant, Campbell \& Kerr } \\
\text { (2003), Klein, Noe \& Wang (2006), Sitzmann } \\
\text { et al. (2006), Al-Shammari (2005), Paechter } \\
\text { \& Maier (2010), Walker (2003), Proserpio \& } \\
\text { Gioia (2007), Rungtusanatham et al. (2004), } \\
\text { Hollenbeck, Zinkhan e French (2005). }\end{array}$ \\
\hline
\end{tabular}

Para situações de aprendizagem cooperativa, o cenário está mais propício a interação por meio da aprendizagem combinada, mas com relação a disseminação de informação, sugere-se a modalidade EaD. Nesse sentido, o instrutor em todos os cenários possui responsabilidade no 
que diz respeito ao aprendizado dos alunos, principalmente quando seu discurso é relevante para o desenvolvimento do conhecimento, visto que a comunicação presencial estabelece uma relação interpessoal positiva, e consequentemente um aspecto da aprendizagem que é importante para a manutenção de motivação de aprendizagem (Price et al., 2007).

Como sugestões de estudos futuros, verificamos a necessidade de abordagens quantitativas na estrutura da comunidade de investigação, para que seja possível examinar elementos destas comunidades, verificando relações e resultados da aprendizagem. Também sugerimos pesquisas relacionadas aos cinco campos instrucionais apresentados por Paechter \& Maier (2010).

\section{Conclusão}

O acoplamento bibliográfico, método utilizado neste estudo, demonstra os crescentes campos de publicação, indicando a frente de pesquisa em um campo (Zupic, \& Čater, 2015), e é útil para detectar tendências e possíveis caminhos para o tema. A principal contribuição do nosso estudo está relacionada a interpretação dessas tendências transformadas em cenários para a EaD em IES, sendo útil para a gestão desse modelo de educação que está em franca ascensão e que no futuro próximo irá sobrepujar o método tradicional de ensino. Outra contribuição relevante demonstrou que nos três cenários apresentados, ficou evidente que a interação é um forte componente para a satisfação dos alunos.

Portanto, para que o EaD seja bem sucedido é necessária que as IES direcionem esforços e invistam na atração e retenção os alunos, bem como em profissionais com competências que ultrapassem os conhecimentos teóricos. Noble (2003) afirma que a educação de qualidade é trabalho intensivo e requer uma baixa relação aluno-professor e significativa interação alunoprofessor, a qual é reafirmada pelos cenários descritos por nós. Qualquer esforço para oferecer qualidade na educação pressupõe um investimento substancial em mão de obra educacional (Rovai \& Downey, 2010).

\section{Agradecimento}

O presente trabalho foi realizado com apoio da Coordenação de Aperfeiçoamento de Pessoal de Nível Superior - Brasil (CAPES) - Código de Financiamento 001. 


\section{Referências}

Abraham, T. (2002). Evaluating the virtual management information systems (MIS) classroom. Journal of Information Systems Education, 13(2), 125-133.

Abrami, P. C., Bernard, R. M., Bures, E. M., Borokhovski, E., \& Tamim, R. M. (2011). Interaction in distance education and online learning: Using evidence and theory to improve practice. Journal of Computing in Higher Education, 23(2-3), 82-103.

Al-Shammari, M. (2005). Assessing the learning experience in a business process reengineering (BPR) course at the University of Bahrain. Business Process Management Journal, 11(1), 4762 .

Anderson, T. D., \& Garrison, D. R. (1995). Critical thinking in distance education: Developing critical communities in an audio teleconference context. Higher Education, 29(2), 183-199.

Anderson, T. (2003). Getting the mix right again: An updated and theoretical rationale for interaction. International Review of Research in Open and Distance Learning, 4(2), 9-14.

Arbaugh, J. B., Bangert, A., \& Cleveland-Innes, M. (2010). Subject matter effects and the community of inquiry $(\mathrm{CoI})$ framework: An exploratory study. The Internet and Higher Education, 13(1), 37-44.

Arbaugh, J. B., Godfrey, M. R., Johnson, M., Pollack, B. L., Niendorf, B., \& Wresch, W. (2009). Research in online and blended learning in the business disciplines: Key findings and possible future directions. The Internet and Higher Education, 12(2), 71-87.

Arbaugh, J. B., \& Warell, S. S. (2009). Distance learning and web-based instruction in management education. In S. J. Armstrong \& C.V. Fukami (Eds.), The SAGE Handbook of Management Learning, education, and development (pp. 231-254). London: SAGE

Publications.

Barnard, L., Lan,W. Y., To, Y. M., Paton, V. O., \& Lai, S. L. (2009). Measuring self-regulation in online and blended learning environments. Internet and Higher Education, 12, 1-6.

Beldarrain, Y. (2008, November). Integrating interaction in distance learning: A comparative analysis of five design frameworks. In E-Learn: World Conference on E-Learning in Corporate, Government, Healthcare, and Higher Education(pp. 1471-1477). Association for the Advancement of Computing in Education (AACE).

Berger, N. S. (1999). Pioneering experiences in distance learning: Lessons learned. Journal of Management Education, 23, 684-690.

Bernard, R. M., Abrami, P. C., Borokhovski, E., Wade, A., Tamim, R., Surkes, M. A. (2009). A meta-analysis of three interaction treatments in distance education. Review of Educational Research, 79(3), 1243-1289. 
Boaventura, J. M. G, Costa, B. K, \& Fischmann, A. (2005) Cenários: metodologia e métodos de construção. In Costa, B. K. \& Almeida, M. I. R. Estratégia: direcionando negócios e organizações. São Paulo: Atlas, 2005. Cap. 1 p.23-44.

Bowen, V. S. (1996). The relationship of locus of control and cognitive style to selfinstructional strategies, sequencing, and outcomes in a learner-controlled multimedia environment. Dissertation Abstracts International Section A: Humanities \& Social Sciences, 56(10-A), 3922 Apr

1996.

Bryant, K., Campbell, J., \& Kerr, D. (2003). Impact of web based flexible learning on academic performance in information systems. Journal of Information Systems Education, 14(1), 41-50.

Bryant, S. M., Kahle, J. B., \& Schafer, B. A. (2005). Distance education: A review of the contemporary literature. Issues in Accounting Education, 20, 255-272.

Broad, M. C. (1999). The dynamics of quality assurance in online distance education. Electronic Journal of Instructional Science and Technology, 3(1), 12-21.

Brophy, J. E. (1999). Teaching: Educational practices series, Vol. 1,

Bruner, J. (1985). Vygotsky: A historical and conceptual perspective. Culture, Communication, and Cognition: Vygotskian Perspectives, 21, 34.

Cook, D. A. (2009). The failure of e-learning research to inform educational practice and what we can do about it. Medical Teacher, 31(2), 158-162.

Cunningham, C. A., \& Billingsley, M. (2003). Curriculum Webs: A practical guide to weaving the Web into teaching and learning. Boston: Allyn and Bacon.

de Almeida, M. E. B. (2003). Educação a distância na internet: abordagens e contribuições dos ambientes digitais de aprendizagem. Educação e pesquisa, 29(2), 327-340.

Daspit, J. J., \& D'Souza, D. E. (2012). Using the Community of Inquiry Framework to Introduce Wiki Environments in Blended-Learning Pedagogies: Evidence From a Business Capstone Course. Academy of Management Learning \& Education, 11(4), 666-683.

Dias, M. A. de P., Vianna, J. N. de S., \& Felby, C. (2016). Sustainability in the prospective scenarios methods: A case study of scenarios for biodiesel industry in Brazil, for 2030. Futures, $82,1-14$.

Ehlers, U. (2004). Quality in e-learning. The learner as a key quality assurance category. European Journal of Vocational Training, 29, 3-15.

Furrer, O. \& Thomas, H. (2000), The rivalry matrix: understanding rivalry and competitive dynamics, European Management Journal, Vol. 18, pp. 619-37.

Garrison, D. R. (1991). Critical thinking and adult education: A conceptual model for developing critical thinking in adult learners. International Journal of Lifelong Education, 10(4), 287-303. 
Garrison, D. R., Anderson, T., \& Archer, W. (2000). Critical inquiry in a text-based environment: Computer conferencing in higher education. The Internet and Higher Education, 2(2), 87-105.

Garrison, D. R., \& Arbaugh, J. B. (2007). Researching the community of inquiry framework: Review, issues, and future directions. The Internet and Higher Education, 10(3), 157-172.

Garrison, D. R., \& Cleveland-Innes, M. (2005). Facilitating cognitive presence in online learning: Interaction is not enough. The American Journal of Distance Education, 19(3), 133-148.

Garrison, D. R., Cleveland-Innes, M., \& Fung, T. S. (2009). Exploring causal relationships among teaching, cognitive and social presence: Student perceptions of the community of inquiry framework. The Internet and Higher Education, 13(1), 31-36.

Georgantzas, N. C., \& Acar, W. (1995). Scenario-driven planning: learning to manage strategic uncertainty. Praeger.

Gunawardena, L., Lowe, C., \& Anderson, T. (1997). Interaction analysis of a global online debate and the development of a constructivist interaction analysis model for computer conferencing. Journal of Educational Computing Research, 17(4), 395-429.

Hagaman, S. (1990). The community of inquiry: An approach to collaborative learning. Studies in Art Education, 31(3), 149-157.

Han, H., \& Johnson, S. D. (2012). Relationship between students' emotional intelligence, social bond, and interactions in online learning. Educational Technology \& Society, 15(1), 78-89.

Harasim, L. (2000). Shift happens: Online education as a new paradigm in learning. Internet and Higher Education, 3(1-2), 41-61.

Heckman, R., \& Annabi, H. (2005). A content analytic comparison of learning processes in online and face-to-face case study discussions. Journal of Computer-Mediated Communication, 10(2).

Herrington, J., Reeves, T. C., \& Oliver, R. (2006). Authentic tasks online: A synergy among learner, task, and technology. Distance Education, 27(2), 233-247.

INEP. (2018) Instituto Nacional de Estudos e Pesquisas Educacionais Anísio Teixeira. Disponível em: 〈http://portal.inep.gov.br/web/guest/inicio>.

Hickey, D., \& McCaslin, M. (2001). A Comparative, socio-cultural analysis of context and motivation. In S. Volet \& S. Ja“rvala“ (Eds.), Motivation in learning contexts: Theoretical advancements and methodological implications (pp. 33-55). Elmsford, NY: Pergamon Press.

Hirumi, A. (2011). The design and sequencing of online and blended learning interactions: A framework for grounded design. Canadian Learning Journal, 16(2), 21-25. 
Hollenbeck, C. R., Zinkhan, G. M., \& French, W. (2005). Distance learning trends and benchmarks: Lessons from an online MBA program. Marketing Education Review, 15(2), 39-52.

Jaspers, F. (1991). Interactivity or instruction? A reaction to Merrill. Educational Technology, $31(3), 21-24$.

Juwah, C. (Ed.). (2006). Interactions in online learning: Implications for theory and practice. New York, NY: Routledge.

Klein, H. J., Noe, R. A., \&Wang, C. (2006). Motivation to learn and course outcomes: The impact of delivery mode, learning goal orientation, and perceived barriers and enablers. Personnel Psychology, 59, 665-702.

Knowles, M. S. (1980). The modern practice of adult education: From pedagogy to andragogy. Englewood Cliffs: Prentice Hall/Cambridge.

Kramarski, B., \& Gutman, M. (2006). How can self-regulated learning be supported in mathematical E-learning environments? Journal of Computer Assisted Learning, 22, 24-33.

Kramarski, B., \& Mizrachi, N. (2006). Online discussion and self-regulated learning: Effects of instructionalmethods onmathematical literacy. The Journal of Educational Research, 99(4), 218-230.

Kuo, Y. C., Walker, A. E., Schroder, K. E., \& Belland, B. R. (2014). Interaction, Internet selfefficacy, and self-regulated learning as predictors of student satisfaction in online education courses. The Internet and Higher Education, 20, 35-50.

Lan,W. Y. (1996). The effects of self-monitoring on students' course performance, use of learning strategies, attitude, self-judgment ability, and knowledge representation. Journal of Experimental Education, 64, 101-115.

Liaw, S. S. (2008). Investigating students' perceived satisfaction, behavioral intention, and effectiveness of e-learning: A case study of the Blackboard system. Computers and Education, 51(2), 864-873.

Lipman, M. (1976). Philosophy for children. Metaphilosophy, 7(1), 17-33.

Lipman, M. (1991). Thinking in education. Cambridge: Cambridge University Press.

Mannermaa, M. (1991). In search of an evolutionary paradigm for futures research. Futures, 23(4), 349-372.

Martins, P. P. P., Boaventura, J. M. G., Fischmann, A. A., Costa, B. K., \& Spers, R. G. (2012). Scenarios for the Brazilian Road Freight Transport Industry. Foresight, 14(3), 207-224.

Molenda, M. (2008). Historical Foundations. In J. M. Spector, M. D. Merrill, J. Elen, \& M. J. Bishop (Eds.), Handbook of Research on Educational Communications and Technology. New York, NY: Springer New York. 
Moore, M. G. (1989). Three types of interaction. American Journal of Distance Education, 3(2), $1-6$.

Moore, M. G. (1993). Theory of transactional distance. In D. Keegan (Ed.), Theoretical principles of distance education (pp. 22-28). New York, NY: Routledge.

Oliveira, A. S., Barros, M. D. de, Pereira, F. de C., Gomes, C. F. S., \& Costa, H. G. da. (2018). Prospective scenarios: A literature review on the Scopus database. Futures, 100, 20-33.

Paechter, M., \& Maier, B. (2010). Online or face-to-face? Students' experiences and preferences in e-learning. The internet and higher education, 13(4), 292-297.

Piccoli, G., Ahmad, R., \& Ives, B. (2001). Web-based virtual learning environments: A research framework and a preliminary assessment of effectiveness in basic IT skills training. MIS Quarterly, 25, 401-426.

Porter, M. E. (1985) Competitive Advantage. New York: Free Press.

Proserpio, L., \& Gioia, D. A. (2007). Teaching the virtual generation. Academy of Management Learning \& Education, 6, 69-80.

Ringland, G. (1998), Scenario Planning - Managing for the Future, John Wiley \& Sons, Chichester.

Rungtusanatham, M., Ellram, L. M., Siferd, S. P., \& Salik, S. (2004). Toward a typology of business education in the Internet age. Decision Sciences Journal of Innovative Education, 2, $101-120$.

Sankaran, S. R., \& Bui, T. X. (2001). Impact of learning strategies and motivation on performance: A study in web-based instruction. Journal of Instructional Psychology, 28, 191-198.

Sankaran, S. R., Sankaran, D., \& Bui, T. (2000). Effect of student attitude to course format of learning performance: An empirical study in web vs. lecture instruction. Journal of Instructional Psychology,

27 , $66-73$.

Schwartz, P. (2000). A arte da visão de longo prazo. São Paulo: Nova Cultural.

Sims, R. (1999). Interactivity on stage: Strategies for learner-designer communication. Australian Journal of Educational Technology, 15(3), 257-272.

Sitzmann, T., Kraiger, K., Stewart, D., \& Wisher, R. (2006). The comparative effectiveness of web-based and classroom instruction: A meta-analysis. Personnel Psychology, 59, 623-664.

Soong, M. . B., Chan, H. C., Chua, B. C., \& Loh, K. F. (2001). Benson Soong et al- (2001) Critical success factors for on-line course resources- Computers and education. Computers \& Education, 36. 
Storopoli, J. E. (2016). O uso do Knowledge discovery in database (KDD) de informações patentárias sobre ensino a distância: contribuições para instituições de ensino superior. Universidade Nove de Julho - UNINOVE. https://doi.org/10.1126/science.1103618

Sun, P. C., Tsai, R. J., Finger, G., Chen, Y. Y., \& Yeh, D. (2008). What drives a successful eLearning? An empirical investigation of the critical factors influencing learner satisfaction. Computers and Education, 50(4), 1183-1202.

Sutton, L. A. (2001). The principle of vicarious interaction in computer-mediated communications. International Journal of Educational Telecommunications, 7(3), 223-242.

Trickey, S., \& Topping, K. J. (2004). "Philosophy for children": a systematic review. Research Papers in Education, 19(3), 365-380.

Vygotsky, L. S. (1978). Mind in society: The development of higher mental process. Cambridge, MA: Harvard University Press.

Wade, W. (2012). Scenario planning: a field guide to the future. New Jersey: Wiley \& Sons Inc.

Wagner, E. D. (1994). In support of a functional definition of interaction. The American Journal of Distance Education, 8(2), 6-29.

Walker, K. (2003). Applying distributed learning theory in online business communication courses. Business Communication Quarterly, 66(2), 55-67.

Wan, Z., Fang, Y., \& Neufeld, D. J. (2007). The role of information technology in technologymediated learning: A review of the past for the future. Journal of Information Systems Education, 18, 183-192.

Young, A., \& Norgard, C. (2006). Assessing the quality of online courses from the students' perspective. The Internet and Higher Education, 9, 107-115.

Zimmerman, B. J. (2008). Investigating self-regulation and motivation: Historical background,methodological developments and future prospects. American Educational Research Journal, 45(1), 166-183. 